\title{
An efficient method for estimating semantic similarity based on feature overlap: Reliability and validity of semantic feature ratings
}

\author{
WILLIAM S. MAKI \\ Texas Tech University, Lubbock, Texas \\ MARISSA KRIMSKY \\ Oklahoma School of Science and Mathematics, Oklahoma City, Oklahoma \\ and \\ SOL MUÑOZ \\ Texas Tech University, Lubbock, Texas
}

\begin{abstract}
Two studies were conducted in which human participants rated pairs of words according to the perceived degree to which the words' referents shared semantic features. The participants found the task intuitive, simple, and quick to complete. The ratings were reliable and valid. Interrater and interstudy correlations were high, and ratings were good predictors of known feature overlap values obtained from existing semantic feature norms.
\end{abstract}

The concept of semantic feature overlap plays a central role in research and theory concerned with semantic memory. The concept is central to models of the computation of meaning (see, e.g., Cree \& McRae, 2003; Cree, McRae, \& McNorgan, 1999; Harm \& Seidenberg, 2004; McRae, de Sa, \& Seidenberg, 1997; Moss, Hare, Day, \& Tyler, 1994; Plaut, 1995). The measurement of semantic feature overlap is theoretically and empirically important, for example, for resolving continuing controversy over the roles played by semantic and associative variables in semantic priming (for reviews, see Hutchison, 2003, and Lucas, 2000).

On the surface, the development of empirical measures of semantic feature overlap appears to depend on knowledge of lists of features associated with various concepts, and such semantic feature norms have been derived using feature-naming procedures (McRae, Cree, Seidenberg, $\&$ McNorgan, 2005). But norms are notoriously costly. It took 12 years to obtain semantic feature norms for 541 concepts (McRae et al., 2005) and many more years to obtain word association norms for 5,019 words (Nelson, McEvoy, \& Schreiber, 1998). ${ }^{1}$ The high costs of obtaining norms have led some to consider computational measures as a substitute (e.g., Church \& Hanks, 1989). The problem with computational measures is that their relationship to normative data obtained from human raters is known

M.K. was supported during the summer of 2004 by the Clark Scholars program at Texas Tech University. Address correspondence to W. S. Maki, Department of Psychology, Texas Tech University, Lubbock, TX 79409 (e-mail: bill.maki@ttu.edu). for only some measures. For example, the reliability and validity of semantic distances computed from the electronic dictionary WordNet (Fellbaum, 1998) have been confirmed in studies of human ratings. Maki, McKinley, and Thompson (2004) used recently developed software (Patwardhan \& Pedersen, 2003) to compute a measure of semantic distance (Jiang \& Conrath, 1997). Human observers were shown to be able to discriminate among such distances. In contrast, Harm (2002) reported a procedure for mining WordNet for semantic features, but the resulting feature lists have not yet been checked against the human feature norms collected by McRae et al. (2005). So, while automatic mining of electronic resources is both computationally feasible and economically desirable, the ultimate value of such measures to cognitive psychologists rests on their correspondence to human-generated norms, and that again raises the issue of the costs of obtaining those norms.

In many applications of normative data in cognitive psychology, it is not necessary to know about the full range of responses to a word or concept. For example, one can select pairs of words on the basis of their association values without knowing about the distribution of all possible responses. Likewise, if one is interested in selecting word pairs on the basis of semantic similarity, then knowing the value of some feature overlap measure between two concepts is sufficient; knowing about the identities of all the features defining those concepts is unnecessary.

The purpose of the research reported here was to explore an alternative method for obtaining measurements of semantic feature overlap. We reasoned that if human subjects can provide lists of features that define concepts, 
then they might also be able to provide estimates of the extent to which any two concepts share features. Ratings of feature overlap might be obtained far more quickly and with less experimental time and computational cost than was the case for the feature-naming procedures used by McRae et al. (2005). In this article, we report our procedures for obtaining such ratings and evaluate their reliability and validity in two studies performed with different groups of participants.

\section{METHOD}

\section{Participants}

Study 1 included a total of 10 volunteers ( 8 females and 2 males). Three females and 1 male were recruited from a summer science program for high school students. Four females were advanced undergraduate psychology majors. The remaining participants (1 male and 1 female) were graduate students. The pool of participants for the first study included the second and third authors. Study 2 included 19 college students ( 14 female, 5 male) recruited from the Texas Tech University human subjects pool; these students, all members of a general psychology course, participated in return for course credit.

\section{Materials and Measures}

McRae et al. (2005) built their semantic feature norms by having many participants list features thought to define a concept; the features included both properties (physical, functional, and categorical) and encyclopedic facts. The result is a large frequency matrix, with each row representing a concept and each column representing a named feature. The cell entries are the frequencies with which each feature was named for a particular concept. A measure of feature overlap for any two concepts is obtained by computing the cosine between the respective row vectors. A cosine of 0 indicates that the concepts are unrelated; a cosine of +1 indicates complete overlap.

In the present studies, pairs of words were selected from the semantic feature norms that had nonzero cosines. Five pairs were selected randomly from each of nine cosine ranges, $0-.1, .1-.2 \ldots$ .8-9. Only 7 pairs appeared in the Maki et al. (2004) version of the association norms; the forward and backward strengths of those pairs are listed in the Appendix. The remaining 38 pairs are not directly associated, by virtue of their absence from the association norms. ${ }^{2}$ Two random orderings of the 45 pairs were created; each was presented to about half of the participants.

Two other measures of semantic similarity were computed for each of the pairs. One measure (JCN) was computed from WordNet. JCN (Jiang \& Conrath, 1997) is a combination measure that is based on both the numbers of nodes separating any two concepts and the information content of the nodes and that of their lowest shared superordinate. Essentially, JCN measures the informational distance between concepts. Maki et al. (2004) showed that JCN is a better predictor of semantic similarity ratings than, for example, an edge-based measure based only on counting nodes. However, Maki et al. also reported that there was considerable unshared variance between JCN and the feature overlap cosine measure of McRae et al. (2005). For the latter reason, the JCN measure was included here; we wanted to know how our feature ratings would fare relative to the JCN measure in predicting the criterion variable (the feature overlap cosines).

The second measure of semantic similarity was the cosine based on latent semantic analyses (LSA; see, e.g., Landauer \& Dumais, 1997). This measure is derived from a large word $\times$ document matrix in which cell entries are based on the frequencies with which words appear in documents. The LSA space was based on the "General Reading up to 1st Year College" corpus, using the computational tools available on the LSA Web site (http://lsa.colorado.edu). As with the JCN measure, we wanted to learn how well LSA would predict feature overlap relative to our human ratings.

An additional 12 pairs were selected for use as training items during the instructions at the beginning of each study. These pairs were selected so as to have low feature overlap (cosine range: .023-.064), medium feature overlap (.492-.546), or high feature overlap (.803.862 ). These pairs are also listed in the Appendix.

\section{Procedure}

The participants were tested in small groups $(n=1-4)$. The participants first read a short essay on how semantic features define concepts. The essay compared features shared and not shared by three animate creatures (a tortoise, a turtle, and a snail) and one inanimate object (a banner). The essay ended with an admonition to rate pairs of words only on shared features and not on other dimensions (associations and taxonomic category). The essay is printed in full in the Appendix. The participants then rated 3 pairs of words (also shown in the Appendix) on a 10-point scale. The instructions were:

Here is an example in which you will judge pairs of words and determine a number on a scale of $0-9$ on how much you think the characteristics of these words overlap, where 0 means no overlap and 9 signifies the words almost mean the same thing. Remember: You should NOT be rating how often the words appear together in context, or their taxonomical similarity.

The pairs were chosen so as to differ markedly in their feature overlap and thus produce wide variations across pairs in the participants' ratings. After they made their ratings, the participants were told about how the pairs differed in feature overlap. Then the participants went on to rate an additional 9 pairs (see Appendix), which also varied considerably in their cosines. Once again, after the ratings were complete, the participants' ratings were compared with the overlap values, and the participants were informed of the known values (the cosines from McRae et al., 2005). The participants then proceeded to the final rating task, in which they rated the 45 test pairs. The entire procedure lasted 15 to $20 \mathrm{~min}$.

\section{RESULTS}

\section{Interrater Reliability}

Study 1. Using the ratings of test pairs, the ratings for each individual rater were correlated with the ratings from every other rater. Each of the product-moment correlations was significant, at better than $p<.05$, the significance level to be used throughout. Summary statistics for these correlations are listed in Table 1. As another check on the reliability of these ratings, we computed the mean interrater reliability for each rater - that is, the average of that individual's correlations with all other raters. These average individual correlations, all significant, ranged from +.805 to +.872 .

Table 1

Summary of Product-Moment Correlations Among Raters

\begin{tabular}{lcccc}
\hline Statistic & Test 1 & Test 2 & Training 1 & Training 2 \\
\hline Median & +.842 & +.741 & +.825 & +.856 \\
Min & +.724 & +.450 & +.403 & +.569 \\
Max & +.913 & +.949 & +.977 & +.994 \\
Mean & +.837 & +.732 & +.810 & +.848 \\
SD & +.045 & +.094 & +.137 & +.084 \\
\hline
\end{tabular}

Note-For test pairs in Studies 1 and 2, $n=45$; for training pairs, $n=$ 12. All correlations are significant at better than the .05 level for the test pairs, and in each study, all but the lowest one of the correlations are significant. 
Study 2. The same analyses were performed on the ratings collected in the second study. One rater was dropped because of exceptionally low correlations, averaging $r=$ .303. Otherwise, all correlations summarized in Table 1 were significant. Also significant were the average individual correlations with every other rater, which ranged from .628 to .800 . So the ratings given by the human subjects pool participants in the second study were less reliable, on average, and the reliabilities themselves were more variable.

Reliability across studies. In spite of the lower reliability in the second study, there was still strong agreement across studies. Ratings were averaged for each pair of words over raters within each study, and we then correlated the average ratings across studies. The significant correlation, $r=+.972$, indicates that the average ratings of both groups of raters were highly reliable. Thus, the aggregate ratings transcended the variations in interrater reliabilities within groups; in spite of the greater variability in the human subjects pool, this group's ratings are quite similar to those of the volunteers in Study 1.

\section{Criterion Validity}

Study 1. Ratings were averaged for each item across raters. The feature overlap cosine was significantly correlated with the mean ratings; the correlations among all measures are shown in Table $2 .{ }^{3}$ The feature overlap cosine (the criterion variable) was predicted by the mean ratings, $\mathrm{JCN}$, and LSA values in a stepwise multiple regression analysis. Only the mean ratings significantly predicted the cosine values $\left[R^{2}=.812, \beta=+.901 ; F(1,43)=185.85\right]$. A separate simultaneous regression that included mean ratings, JCN, and LSA as predictors confirmed that JCN and LSA were not reliable predictors. JCN and LSA accounted only for an additional $0.4 \%$ of the variance in cosines, and $R^{2}$ only increased from +.812 to +.816 $(\beta=+.82,-.076$, and .039 for mean ratings, JCN, and LSA, respectively) $\left[F_{\text {change }}(2,41)<1\right]$.

Study 2. Ratings were again averaged for each pair of words across participants. As in Study 1, feature overlap cosine and the mean ratings were significantly correlated (see Table 2). Mean ratings and JCN were predictor variables, and feature overlap cosine was the criterion variable in multiple regression analyses. In the stepwise analysis, only the mean ratings entered significantly, accounting for nearly $80 \%$ of the variance in cosines $\left[R^{2}=.784\right.$, $\beta=+.885 ; F(1,43)=155.72]$. The simultaneous regression showed that JCN and LSA did not add significantly

Table 2 Summary of Product-Moment Correlation Measures

\begin{tabular}{lcccc}
\hline & Cosine & Rating & JCN & LSA \\
\hline Cosine & - & .901 & -.707 & .588 \\
Rating & .885 & - & -.743 & .620 \\
JCN & -.707 & -.719 & - & -.529 \\
LSA & .588 & .612 & -.529 & - \\
\hline
\end{tabular}

Note-Correlations above the diagonal are from Study 1; correlations below the diagonal are from Study 2. All correlations are significant. See text for definitions of measures. to the explained variance in cosines, only increasing $R^{2}$ by $.012(\beta=+.755,-.135$, and .055 for mean ratings, JCN, and LSA, respectively) $\left[F_{\text {change }}(2,41)=1.20\right]$.

\section{Training Data}

The high degree of reliability and validity observed in these studies for the test pairs was also found for the training pairs. The interrater correlations are summarized in Table 1. As with the test pairs, the average ratings for each pair were averaged across raters in each study. The correlation between the average ratings across studies was $r=.986$. Average ratings were correlated with the cosines for all 12 training pairs. For Study $1, r=.950$, and for Study $2, r=.956$.

\section{DISCUSSION}

Our first question was whether people could rate pairs of concepts based on their degree of shared semantic features - that is, semantic feature overlap. From the very start of this research, it was apparent that our participants found this to be an intuitive and simple task. There was remarkable agreement among raters at the outset of our "training" as to which training pairs shared many, some, or few features. Our second question concerned the reliability of such ratings. In both training and test phases of both our studies, there was a high degree of interrater reliability. Moreover, in spite of the differences in the participants in our two studies, the reliability across studies was extremely high (over .97 for both test and training phases). Our third question was whether such ratings were valid - that is, predictive of the cosine measure of feature overlap obtained from the McRae et al. (2005) feature norms. Regression analyses showed that the mean rating (per item) in each study accurately predicted the cosines and did so far better than the other semantic similarity measures-JCN, derived from WordNet, and the LSA cosine. In fact, these two measures added nothing significant to the explained variance in cosines beyond what was attributed to the mean feature ratings. Thus, we have shown that people can judge semantic overlap and that their judgments are reliable and valid.

It might be noted that the correlations we obtained between feature overlap ratings and the cosine measure from the feature-naming norms are much larger than those previously reported by McRae et al. (1997). In their Experiment 2B, they collected ratings of similarity of pairs of words and reported correlations between similarity ratings and cosines of $.57\left(r^{2}=.33\right)$ and lower. Our correlations may be so much higher because of differences in the pairs of words we used. But the correlation may also be influenced by the specificity of the instructions. We were quite explicit in asking our participants to rate on the basis of shared features, thus providing them with a specific definition of "similarity."

The regression analyses reported in the text focused on establishing the criterion validity of the feature overlap ratings; consequently, the feature cosine is the variable predicted by ratings (and other measures). We performed 
another set of analyses, in which the dependent variable was the feature overlap rating and the predictor variables were the feature cosine, JCN, and LSA scores. The results paralleled those reported in the Results; the feature cosine accounted for about $80 \%$ of the variance in ratings, with the JCN and LSA variables accounting for around $2 \%$. These results supplement those recently reported by Vigliocco, Vinson, Lewis, and Garrett (2004). They derived a semantic distance measure based on feature-naming procedures (see, e.g., McRae et al., 2005). That distance measure predicted performance in a variety of tasks (picture naming, picture-word interference, and semantic priming) better than did either LSA or a measure of distance in WordNet. The relationship between our feature ratings and the Vigliocco et al. semantic distance measure remains to be determined, but, on the basis of the research reported here, we expect the correlation between the two measures to be high.

The semantic feature-rating procedure is efficient. Our raters completed ratings for 45 pairs in no more than $20 \mathrm{~min}$ (roughly averaging $0.5 \mathrm{~min}$ per pair). McRae et al. (2005) report 40-50 min spent on 20-24 concepts, thus averaging roughly $2 \mathrm{~min}$ per concept. The cost of obtaining overlap ratings relative to that for obtaining feature norms is reduced further when the numbers of participants are taken into account (10-18 vs. 30). And these investment figures do not take into account the data management and computational costs of single numbers versus lists of strings. ${ }^{4}$ It is our hope that we, and others, in the future, may be able to use the feature-rating method to create a large-scale database of semantic similarity norms based on feature overlap ratings. The resulting database should be useful in the ongoing theoretical and empirical efforts to understand the representation of semantic knowledge.

\section{REFERENCES}

Church, K. W., \& Hanks, P. (1989). Word association norms, mutual information, and lexicography. Proceedings of the 27th Annual Meeting of the Association for Computational Linguistics (pp. 76-83). Vancouver, BC: Association for Computational Linguistics.

Cree, G. S., \& McRae, K. (2003). Analyzing the factors underlying the structure and computation of the meaning of chipmunk, cherry, chisel, cheese, and cello (and many other such concrete nouns). Journal of Experimental Psychology: General, 132, 163-201.

Cree, G. S., McRae, K., \& McNorgan, C. (1999). An attractor model of lexical conceptual processing: Simulating semantic priming. Cognitive Science, 23, 371-414.

Fellbaum, C. (Ed.) (1998). WordNet: An Electronic Lexical Database. Cambridge, MA: MIT Press. Available at wordnet.princeton.edu.

HARM, M. W. (2002). Building large scale distributed semantic feature sets with WordNet (Technical Report PDP.CNS.02.1). Pittsburgh, PA: Carnegie Mellon University, Center for the Neural Basis of Cognition.
Harm, M. W., \& Seidenberg, M. S. (2004). Computing the meanings of words in reading: Cooperative division of labor between visual and phonological processes. Psychological Review, 111, 662-720.

Hutchison, K. A. (2003). Is semantic priming due to association strength or feature overlap? A microanalytic review. Psychonomic Bulletin \& Review, 10, 785-813.

JianG, J. J., \& ConRath, D. W. (1997). Semantic similarity based on corpus statistics and lexical taxonomy. Proceedings of the 10th International Conference on Research in Computational Linguistics (ROCLING X) (pp. 19-33). Taipei: Academica Sinica.

Landauer, T. K., \& Dumais, S. T. (1997). A solution to Plato's problem: The latent semantic analysis theory of acquisition, induction, and representation of knowledge. Psychological Review, 104, 211-240.

LuCAS, M. (2000). Semantic priming without association: A metaanalytic review. Psychonomic Bulletin \& Review, 7, 618-630.

Maki, W. S., McKinley, L. N., \& Thompson, A. G. (2004). Semantic distance norms computed from an electronic dictionary (WordNet). Behavior Research Methods, Instruments, \& Computers, 36, 421431.

McRae, K., Cree, G. S., Seidenberg, M. S., \& McNorgan, C. (2005). Semantic feature production norms for a large set of living and nonliving things. Behavior Research Methods, Instruments, \& Computers, 37, 547-559.

McRae, K., de Sa, V. R., \& Seidenberg, M. S. (1997). On the nature and scope of featural representations of word meaning. Journal of Experimental Psychology: General, 126, 99-130.

Moss, H. E., Hare, M. L., DAy, P., \& Tyler, L. K. (1994). A distributed memory model of the associative boost in semantic priming. Connection Science, 6, 413-427.

Nelson, D. L., McEvoy, C. L., \& Schreiber, T. A. (1998). The University of South Florida word association, rhyme, and word fragment norms. Available at w3.usf.edu/FreeAssociation.

Patwardhan, S., \& Pedersen, T. (2003). WordNet::Similarity. Available at search.cpan.org/dist/WordNet-Similarity.

Plaut, D. C. (1995). Semantic and associative priming in a distributed attractor network. Proceedings of the 17th Annual Conference of the Cognitive Science Society, 17, 37-42.

Vigliocco, G., Vinson, D. P., Lewis, W., \& Garrett, M. F. (2004). Representing the meanings of object and action words: The featural and unitary semantic space hypothesis. Cognitive Psychology, 48, $422-488$.

\section{NOTES}

1. The degree of overlap among these two sets of norms is small, making stimulus selection based on semantics and association difficult.

2. The analyses to be reported included all 45 test pairs. In another analysis, the associated pairs were dropped; the pattern of results was identical to that reported in the Results.

3. Some of the correlations reported in Table 2 are substantially higher than those reported by Maki et al. (2004). Sample size accounts for the difference. Maki et al. included in their analyses all possible pairs of concepts $(n=132,355)$ from the feature-naming norms. In the present study, only 45 pairs were sampled from feature cosine strata, thus minimizing the influence of many pairs with low cosines. But the important correlations are those between feature ratings and the cosines in Table 2; the high correlations (around .9) establish the criterion validity of the feature ratings.

4. The costs of the two procedures are relative to the goals. If the goal is to obtain measures of similarity (feature overlap), then the featurenorming procedure is more costly. But if the goal is to identify semantic features (e.g., for use in simulations of distributed processing models), then feature norming is necessary. 


\section{APPENDIX \\ Instructions Read by Participants}

This experiment is concerned with how humans perceive similarities between the characteristics which define words. Consider the word (and concept of) TORTOISE. We know that a TORTOISE is a reptile with an exoskeleton and a hard shell. If we compare the word TORTOISE with the word TURTLE, we find that they share a majority of the same features. Therefore, their definitions or characteristics overlap greatly.

When compared, the words TORTOISE and SNAIL are found to have characteristics that are fairly similar, but there are almost as many dissimilar traits as there are similar. For example, both animals have exoskeleton shells. However, a tortoise is a reptile and a snail is a mollusk.

This means that the characteristics only moderately overlap. Although TORTOISE and SNAIL share many of the same taxonomy traits, the psychological concepts shared by TORTOISE and SNAIL differ from the taxonomical traits in the sense that the psychological traits separate the two concepts more than taxonomy would.

If we compare the word TORTOISE with the word BANNER, we find that there is very little which these two objects have in common. TORTOISE and BANNER are so dissimilar because the concepts of what makes a TORTOISE and what makes a BANNER hardly overlap at all. For example, they are both physical objects, but a TORTOISE is animate and a BANNER is inanimate.

Words differ along many dimensions. Words are often associated in print and speech (like SHEET-ROCK or CAPROCK) even though they have nothing in common. Some words belong to common categories (TORTOISE and SNAIL both have exoskeletons). However, please try to ignore these relationships between words. In this experiment, your ratings of pairs of words should be based $\mathrm{ONLY}$ on how many features the words share.

Table A1

Some Characteristics of the Word Pairs Used in Studies 1 and 2

\begin{tabular}{|c|c|c|c|c|c|c|c|c|}
\hline Word Pair & $\mathrm{JCN}$ & Cosine & Word Pair & $\mathrm{JCN}$ & Cosine & Word Pair & $\mathrm{JCN}$ & Cosine \\
\hline \multicolumn{9}{|c|}{ Practice Pairs I } \\
\hline catfish-trout & 5.386 & .803 & dog-donkey & 10.012 & .549 & fence-pelican & 20.088 & .034 \\
\hline \multicolumn{9}{|c|}{ Practice Pairs II } \\
\hline blackbird-stork & 10.695 & .522 & chipmunk-coconut & 22.358 & .064 & guppy-minnow & 8.688 & .862 \\
\hline kite-pier & 20.545 & .062 & finch-nightingale & 5.968 & .854 & blender-sled & 19.116 & .028 \\
\hline alligator-crocodile & 1.992 & .822 & peach-prune & 10.922 & .503 & bear-walrus & 11.457 & .492 \\
\hline \multicolumn{9}{|c|}{ Test Pairs } \\
\hline airplane-bull & 16.582 & .075 & bagpipe-saxophone & 7.474 & .509 & beetle-cockroach & 8.038 & .724 \\
\hline belt-ruler & 15.940 & .016 & bison-platypus & 14.707 & .387 & *bracelet-necklace & 6.039 & .616 \\
\hline broom-tomahawk & 19.093 & .163 & cabin-chair & 16.185 & .229 & *cabinet-cupboard & 4.057 & .835 \\
\hline cantaloupe-pear & 9.957 & .451 & cape-rattlesnake & 19.598 & .196 & caribou-moose & 7.188 & .806 \\
\hline cat-otter & 5.975 & .430 & cello-guitar & 8.010 & .632 & cod-tuna & 6.400 & .751 \\
\hline colander-strainer & 4.543 & .789 & *couch-sofa & 0.000 & .879 & cucumber-pickle & 21.248 & .485 \\
\hline *cushion-pillow & 0.657 & .680 & door-pistol & 13.433 & .064 & dove-oriole & 8.884 & .725 \\
\hline eagle-swan & 8.119 & .334 & garlic-seagull & 21.355 & .103 & goat-pig & 5.224 & .395 \\
\hline goldfish-perch & 10.819 & .569 & gopher-shrimp & 16.069 & .151 & grape-tomato & 8.623 & .616 \\
\hline grapefruit-lemon & 4.323 & .739 & hammer-spear & 11.587 & .146 & harp-racquet & 17.756 & .316 \\
\hline harpoon-machete & 23.570 & .549 & knife-pin & 11.674 & .296 & mackerel-salmon & 8.930 & .851 \\
\hline mandarin-pineapple & 9.546 & .546 & mat-radish & 20.803 & .058 & pan-rake & 11.304 & .230 \\
\hline peg-spatula & 14.832 & .341 & pheasant-pigeon & 5.281 & .667 & pie-seaweed & 21.365 & .205 \\
\hline plum-raisin & 9.418 & .488 & *raspberry-strawberry & 6.842 & .849 & rat-rock & 17.999 & .024 \\
\hline${ }^{*}$ ship-yacht & 5.832 & .442 & *shovel-spade & 0.713 & .519 & tuba-whale & 21.714 & .249 \\
\hline
\end{tabular}

Note-JCN is the semantic distance computed from the electronic dictionary WordNet (Maki et al., 2004). Cosine is the measure of feature overlap from the semantic feature norms (McRae et al., 2005). The seven starred test pairs also appear in the word association norms (Maki et al., 2004). Their forward (backward) strengths, listed in the order in which the pairs appear in the table, are $.095(.154), .048(.151), .189(.507), .194(.030), .135(.035), .000(.079)$, and $.021(.155)$. The practice pairs were presented in the order listed (left to right, top to bottom); the test pairs (listed alphabetically) were presented in one of two random orders.

(Manuscript received November 5, 2004 revision accepted for publication March 25, 2005.) 